\title{
Three-dimensional correlative imaging of a malaria-infected cell with a hard $\mathrm{X}$-ray nanoprobe
}

\author{
Y. Yang, ${ }^{*, \dagger}$ F. Fus, ${ }^{\dagger}$ A. Pacureanu, ${ }^{\dagger}$ J. Cesar da Silva, ${ }^{\dagger}$ W. De Nolf, ${ }^{\dagger}$ C. Biot,$^{\ddagger}$ \\ S. Bohic, ${ }^{*, \dagger}$, and P. Cloetens ${ }^{*, \dagger}$ \\ $\dagger E S R F$, the European Synchrotron, Grenoble, France \\ $\ddagger$ Université des Sciences et Technologies de Lille 1, Lille, France \\ I Université Grenoble Alpes, EA-7442 Rayonnement Synchrotron et Recherche Médicale, \\ Grenoble, France \\ E-mail: yang.yang@esrf.eu; bohic@esrf.eu; cloetens@esrf.eu
}

\section{Abstract}

Benefiting from the recent advances of synchrotron X-ray nanoprobes, we demonstrate three-dimensional (3D) correlative nanoimaging on malaria-infected human red blood cells. By combining X-ray fluorescence tomography and phase contrast nanotomography on the same cell with sub-100 nm pixel size, we establish a routine workflow from the data acquisition, data processing to tomographic reconstruction. We quantitatively compare the elemental volumes obtained with different reconstruction methods, the total variation minimization giving the most satisfactory results. We reveal elemental correlations in different cell compartments more reliably on reconstructions as opposed to $2 \mathrm{D}$ projections. Finally, we determine for the first time the 3D mass fraction maps of multiple elements at sub-cellular level. The estimated total number of Fe atoms and the total mass of the red blood cell show very good agreement with previously reported values.

\section{Introduction}

Living systems use and release major, minor and trace elements for survival. Several essential ions participate in the control of numerous metabolic and signaling pathways. In addition, a number of sophisticated networks of trafficking pathways are available to tightly regulate their uptake, intracellular transport and compartmentalization. ${ }^{1}$ The detailed study of these essential elements is a critical step towards a better understanding of their homeostasis or their dysfunctions.

Synchrotron X-ray fluorescence microscopy is a label-free, quantitative and sensitive imaging technique. ${ }^{2-4}$ It contributes to elucidate the metallome through the determination of the elemental distribution, concentration and chemical state of metals inside tissues and cells at the organelle level. However, most of the applications use 2D imaging whereas biological specimens are inherently 3D objects. Therefore, the investigation of the elemental 3D distribution across the sub-cellular complex and interconnected structures is necessary. It can provide the key information to decipher metal compartmentalization and translocation. The advent of high energy X-ray nanoprobes ${ }^{5,6}$ with high flux is opening new possibilities for single cell 3D elemental imaging. The spatial resolution has dramatically improved, but also the sensitivity and the efficiency have increased. Hence more demonstrations and applications are reporting sub-micron X-ray fluorescence nanotomography. ${ }^{7,8}$

The emerging approach of correlative imag- 
ing, which combines X-ray fluorescence tomography and $\mathrm{X}$-ray phase contrast imaging opens new perspectives in bioimaging. ${ }^{9-11}$ It complements qualitatively the elemental information with structural information. Previous works have successfully achieved the absolute quantification in 2D X-ray fluorescence imaging. ${ }^{12,13}$ However, combining the information for improved 3D quantification in terms of mass fractions remains challenging, mainly due to barriers in fluorescence tomography data acquisition and data processing.

Here we exemplified the quantitative use of a correlative 3D imaging workflow on human red blood cells infected with the malaria parasite P. falciparum. The X-ray fluorescence tomography acquisition is optimized in spatial and temporal resolution compared to existing work and complemented with efficient X-ray holographic nanotomography for 3D mapping of the electron density. A workflow for data processing was established, optimizing the fluorescence tomography reconstruction procedure among different methods. Finally we also registered the fluorescence and the phase contrast tomography data to obtain 3D mass fraction maps of the elements. The quantification of the total number of $\mathrm{Fe}$ atoms and the total mass of the cell agreed very well with the values reported in. ${ }^{14-16}$

\section{Experimental}

\section{Data acquisition}

The data acquisition was performed at the ID16A Nano-Imaging beamline of the ESRF, providing an exceptionally bright $\mathrm{X}$-ray nanofocus of $27 \mathrm{~nm}(\mathrm{H}) \times 37 \mathrm{~nm}(\mathrm{~V})$ with a flux of $4 \times 10^{11} \mathrm{ph} / \mathrm{s}$ at $17 \mathrm{keV}$. X-ray holographic nanotomography, ${ }^{17}$ providing the $3 \mathrm{D}$ electron density distribution, was performed first as it requires less dose and is therefore less damaging. For efficient phase retrieval, tomography scans at four different focus-to-sample distances were acquired as illustrated in the center of Fig. 2. Each tomography scan consisted of 1200 projections over $180^{\circ}$ with an exposure time of $0.2 \mathrm{~s}$.

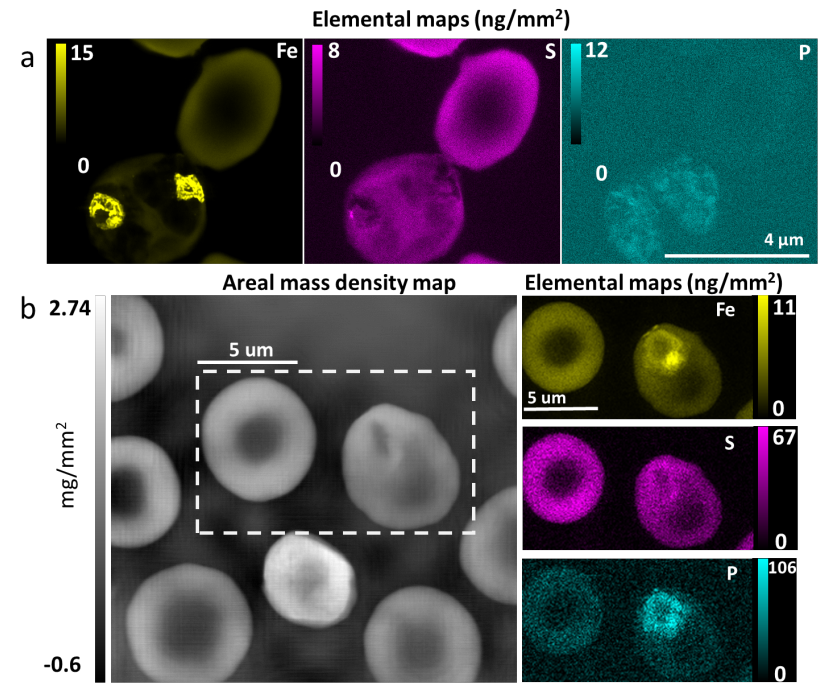

Figure 1: (a) Elemental maps (Fe, S and P) of two red blood cells measured with X-ray scanning fluorescence imaging (unit: $\mathrm{ng} / \mathrm{mm}^{2}$ ), with a step size $30 \mathrm{~nm}$. (b) Left: Areal mass density map (unit: $\mathrm{mg} / \mathrm{mm}^{2}$ ) of the red blood cells retrieved by holographic phase contrast imaging. Right: Elemental maps (unit: $\mathrm{ng} / \mathrm{mm}^{2}$ ) of the same cells boxed in the areal mass density map, measured with a moderate step size of $75 \mathrm{~nm}$.

The effective pixel size of the retrieved phase maps was $10 \mathrm{~nm}$, one of which is shown on the left of Fig. 1(b).

Then the X-ray fluorescence tomography, providing the $3 \mathrm{D}$ elemental concentrations, ${ }^{18}$ was performed by scanning the sample on-the-fly through the X-ray nanofocus at different angular positions. As illustrated in the center of Fig. 2, the X-ray fluorescence emission was collected by a pair of six-element silicon drift detectors (Rayspec, UK) positioned perpendicular to the beam path on each side of the sample. Fig. 1(a) shows high resolution 2D fluorescence elemental maps obtained with a step size of $30 \mathrm{~nm}$. One healthy red blood cell (top right) and another infected by the malaria parasite (bottom left) can be recognized. Different compartments, such as the hemozoin crystals and the digestive vacuole, are clearly differentiated in the Fe map of the infected cell. This very high spatial resolution can unfortunately not be preserved in the 3D fluorescence mapping due to limitations in acquisition time and 
anticipated radiation damage. A compromise between the field-of-view, the step size and the number of angular projections has to be made. In our case, a field-of-view of $15 \mu \mathrm{m} \times 8 \mu \mathrm{m}$ was mapped with a step size of $75 \mathrm{~nm}$ and $50 \mathrm{~ms}$ dwell time, repeated at 22 angles covering a range of $156^{\circ}$ and leaving a missing-wedge of $24^{\circ}$ due to the sample support. This resulted in a total acquisition time for fluorescence tomography of approximately 10 hours including the overhead. Compared to the previously reported experiment duration, such as 36 hours including the overhead in, ${ }^{7} 72$ hours including the overhead in ${ }^{11}$ and 15 hours excluding the overhead in , ${ }^{10}$ this improvement in acquisition time is crucial to perform 3D fluorescence mapping more routinely. Using a beam attenuation by a factor 24 , the total dose delivered to the sample during the fluorescence tomography acquisition was estimated to be $7 \times 10^{8}$ Gy (see Supporting Informtion). ${ }^{19}$

\section{Data processing and tomographic reconstruction}

The processing of the X-ray phase contrast nanotomography datasets follows a well established procedure in two steps: phase retrieval and tomographic reconstruction. The phase retrieval exploits the linearity between the Fourier transform of the images and the Fourier transform of the phase map through the contrast transfer function model. ${ }^{20}$ It is implemented in inhouse code using the GNU Octave language. The phase maps are projections of the refractive index decrement $\delta$ that is proportional to the electron density, and to a good approximation to the total mass density. A 2D projection phase map can thus be converted to the areal mass density as in Fig. 1(b). The second step involves tomography reconstruction. This was performed using the PyHST software developed at ESRF ${ }^{21}$ applying a GPU accelerated implementation of the classical filtered backprojection (FBP) inversion algorithm. ${ }^{22,23}$ The resulting refractive index distribution is converted in local mass density using the Guinier approximation. ${ }^{24}$

The processing pipeline of the fluorescence nanotomography is complex and less established than the one for phase contrast tomography. Briefly, the raw fluorescence spectra, summed over the different detector elements, were fitted using PyMCA, ${ }^{25}$ and calibrated with a thin film reference sample (AXO DRESDEN GmbH) to obtain the areal mass density maps of the different elements. The maps were corrected for a small amount of shrinkage (maximum 5\%) over the acquisitions. A preliminary rough alignment of the sum spectrum projections with a basic cross-correlation algorithm was followed by horizontal fine tuning using the center of mass. The subsequent tomographic reconstruction was realized with the TomoJ plugin from the ImageJ software ${ }^{26}$ for the following algorithms: Simultaneous Iterative Reconstruction Technique (SIRT), ${ }^{27}$ Total Variation Minimization (TVM), ${ }^{28}$ and TVM with positivity constraint. Maximum likelihood expectation minimization (MLEM) ${ }^{29}$ reconstruction was made with XRDUA software, ${ }^{30}$ but is also available in TomoPy. ${ }^{31}$

$3 \mathrm{D}$ volume registration between the elemental mass concentration volumes and the mass density volume is made with $3 \mathrm{D}$ affine registration using the DIPY package. ${ }^{32,33}$ This registration uses mutual information and a multi-resolution strategy to avoid local optima. For absolute quantification, we obtained the 3D mass fraction distributions by a regularized division ${ }^{13}$ of the elemental distributions by the mass density distribution.

\section{Results and discussion}

Fig. 1(b) shows the areal mass density of Fe, $\mathrm{S}$ and $\mathrm{P}$ in two red blood cells (right) and the areal total mass density with a larger field-ofview covering several cells (left). These 2D maps are the multi-modal projections at normal incidence of the tomography datasets. The cell on the left was non-infected, while the one on the right was infected by the malaria $P$. falciparum parasite. In the non-infected cell, the areal mass density maps of Fe, S and P appear similar and proportional to the areal mass density map on the left. This indicates very homo- 


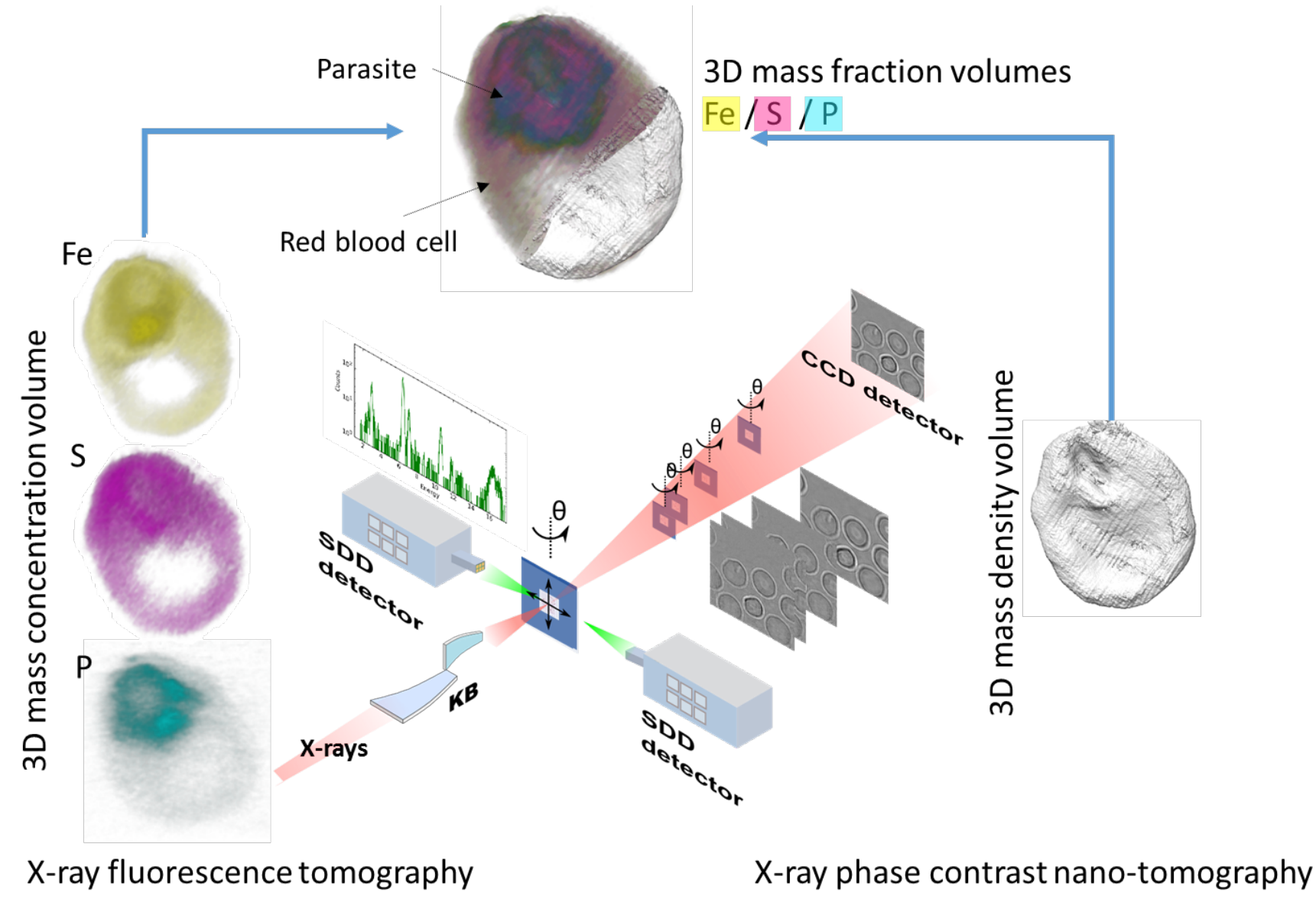

Figure 2: Workflow of correlative imaging with X-ray fluorescence tomography and phase contrast nanotomography to produce 3D mass concentration, mass density and mass fraction volumes. The sample was first put downstream of the X-ray nanoprobe to perform phase contrast nanotomography. The 3D mass density volume is obtained as a result of the tomographic reconstruction (on the right). Subsequent X-ray fluorescence scanning measurements were performed with the same sample in the focus of the nanoprobe. Tomographic reconstruction yields the 3D mass concentration volumes of different elements (on the left). The two multi-modal volumes were then registered to yield 3D mass fraction volumes (top).

geneous distributions of most chemical elements across the whole cellular body. In the infected cell however, one can observe an Fe rich area identified as the parasitic vacuole. Through the digestion of hemoglobin and crystallization of hemozoin, the parasite induces the relocation of Fe inside the cell leading to an intense Xray fluorescence signal typical of the hemozoin crystals. ${ }^{34}$ Interestingly, an increased areal density of $\mathrm{P}$ proved to be associated to the parasitic vacuole. This was also observed in a previous study with electron-probe X-ray microanalysis (EPXMA) where the $\mathrm{P}$ content was higher in the malaria-infected red blood cells than in non-infected cells, showing elevated levels of $\mathrm{P}$ especially in the cytoplasm of the parasites. ${ }^{35}$ This can be interpreted as a sign for increased metabolic activity.

\section{Tomographic reconstruction opti- mization}

One of the goals of the present work is to optimize the quantitative tomographic reconstruction given the challenges set by this type of datasets. The limitations originate from the small number of projections available (22 projections spanning $156^{\circ}$ ), the considerable missing wedge $\left(24^{\circ}\right)$, and the poor signal-to-noise ratio (SNR) for most elements (with the notable 

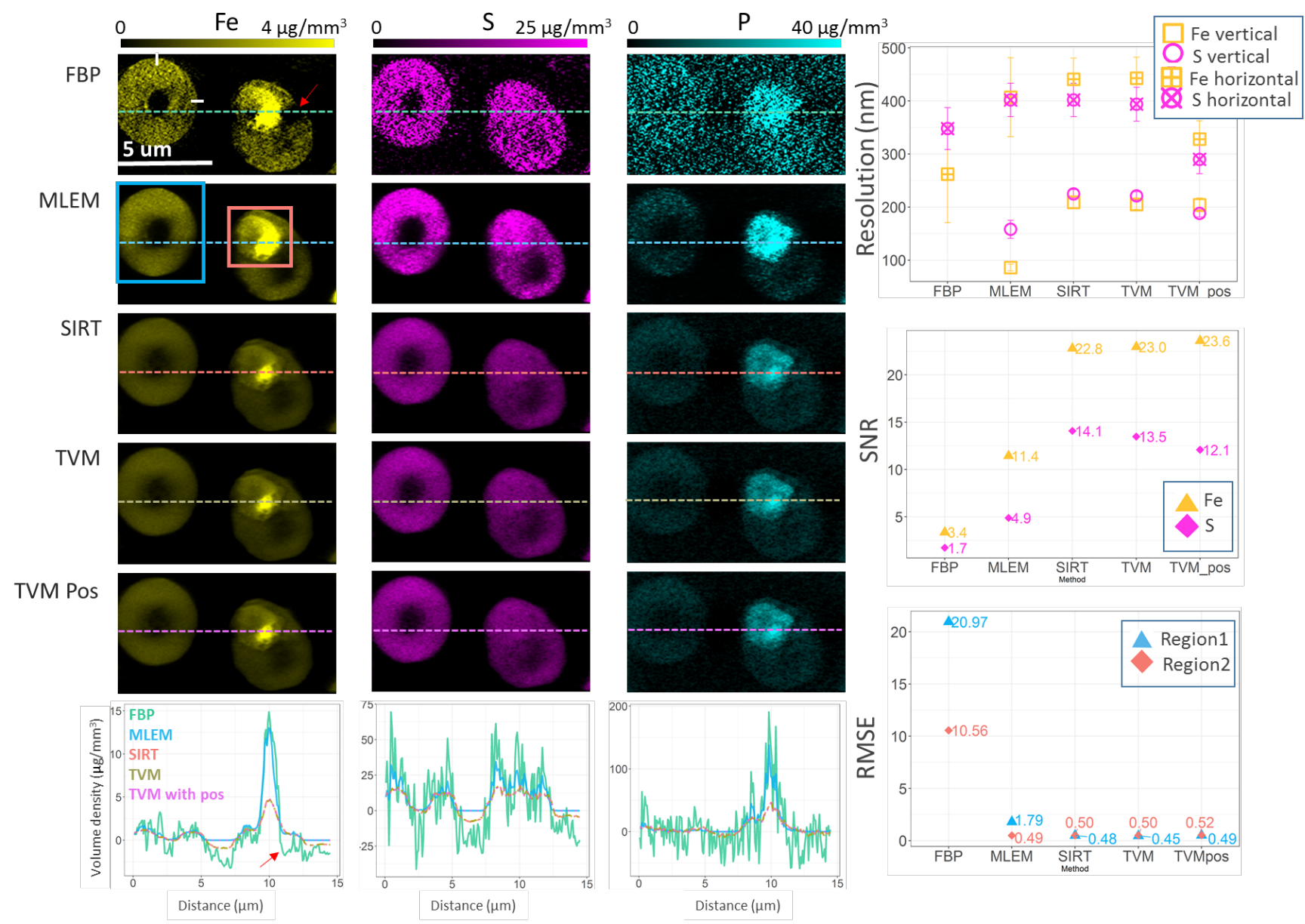

Figure 3: Comparison of the central slice of the tomographic volumes obtained with five reconstruction methods (FBP, MLEM, SIRT, TVM and TVM with positivity constraint). We exemplified with the elements $\mathrm{Fe}, \mathrm{S}$ and $\mathrm{P}$, which are of main biological relevance. The same color bar (unit: $\mu \mathrm{g} / \mathrm{mm}^{3}$ ) applies to the mass concentration obtained with the five methods. Note that the FBP images are saturated in some parts due to their high noise level. Line profiles along the central line of the slices are compared below the column of each element. The plots on the right compare respectively the resolution, signal-to-noise ratio and fidelity of the reconstructions.

exception of $\mathrm{Fe}$ ). This is a common situation for X-ray fluorescence tomography in a biological context. The angular undersampling is the main limitation and becomes the driving factor in the choice of the reconstruction method. We found iterative approaches with regularization to yield the best results.

Fig. 3 summarizes the comparison of the different tomography reconstruction methods. Our analysis were done on the 3D maps of Fe, $\mathrm{S}$, and $\mathrm{P}$ using as comparison metrics: the fullwidth-half-maximum (FWHM) of edge-cutting lines, the SNR and a fidelity evaluation. The FWHM was extracted for $\mathrm{Fe}$ and $\mathrm{S}$ from respectively the horizontal and vertical line indi- cated in white in the first row of the Fe column. Five horizontal and five vertical line profiles were fitted with hyperbolic functions to extract estimates of the resolutions. The standard deviations of the five measurements were used to compare the consistency and spot artifacts. The SNR was defined as ${ }^{36}$

$$
\mathrm{SNR}=\frac{\overline{S_{c e l l}}-\overline{S_{b g}}}{\sqrt{\sigma_{c e l l}{ }^{2}+\sigma_{b g}{ }^{2}}}
$$

where $\overline{S_{\text {cell }}}$ and $\overline{S_{b g}}$ is the average signal in homogeneous regions within cells and the background, respectively. $\sigma_{\text {cell }}$ and $\sigma_{b g}$ denote the standard deviations in the respective regions. 
In fidelity evaluation, the two boxed areas in the Fe column in the second row were used as representative of smooth features (blue box) and non-smooth features (pink box). To evaluate the errors in the reconstructed volumes, we subtracted the sum of reconstructed slices from the corresponding projection map and calculated the root-mean-square-error (RMSE) of the five reconstruction methods.

Although FBP is the fastest deterministic method, it does not provide satisfactory results for our data. It suffers from the angular undersampling and the low SNR in the projection data. As shown in the line profile and the SNR evaluations, high level of noise and large fluctuations hindered the quantification in the reconstructed volumes. Moreover, the missing wedge leads to evident artifacts, illustrated by the red arrow in Fig. 3.

MLEM provides enhanced image quality as a probabilistic based iterative method. It was able to reduce the statistical artifacts without excessive smoothing. Starting from a guess of homogeneous distribution, MLEM restores first the low frequencies and then the high frequencies iteratively. It converged very quickly (10 iterations), but exhibits a high variance that increases with the number of iterations. There is a large discrepancy in the vertical and horizontal resolution values, because with a fixed number of iterations the vertical features of the cell got sharp, while the horizontal features were still blurred. Notwithstanding, MLEM seemed to behave better in regions that are not piecewise constant such as the pink area boxed in Fig. 3. In this area with the hemozoin crystals, the large intensity fluctuations are more prone to be affected by the total variation minimization.

The SIRT and TVM algorithms showed both good denoising efficiency and gave very similar results. This similarity should be related to the small TVM regularization weight used (0.05 in TomoJ). These methods outperformed FBP and MLEM in terms of resolution, SNR and fidelity evaluation. The best FWHMs of $188 \mathrm{~nm}$ in vertical and $290 \mathrm{~nm}$ in horizontal direction were given by TVM with positivity constraint, as it has the advantage of forcing the background to be zero. As a result, we applied TVM with positivity constraint to reconstruct the final elemental volumes.

\section{Quantitative analysis on mass con- centration and mass fraction}

We have quantitatively calculated the elemental mass concentrations, mass fractions and total mass for different cell compartments in Table 1. The parasite to cell ratios in mass fraction are 1.84 for Fe, 1.33 for $\mathrm{S}$ and 2.01 for P. It indicates the uptake percentage of these elements inside the parasitic vacuole. It is also consistent with the observations on the elemental distributions, where $\mathrm{P}$ showed the strongest contrast between the parasite region and the rest of the cell body. The total number of $\mathrm{Fe}$ atoms was calculated to be $9.2 \times 10^{8}$ and $8.9 \times 10^{8}$, respectively in the non-infected and infected red blood cell, in very good agreement with the value $1.08 \times 10^{9}$ in ref, ${ }^{14}$ also consistent with the measurements with vitrified malaria-infected red blood cells. ${ }^{15}$ The total mass of the infected red blood cell of $27.4 \mathrm{pg}$ agreed very well with the value $(27.2 \pm$ $5.3 \mathrm{pg})$ reported in.$^{16}$

Fluorescence tomography of single cells provides the intracellular elemental distributions in $3 \mathrm{D}$, thus their colocalization can be determined unambiguously in contrast with 2D scanning for which elemental correlations are often obscured by overlaps. The scatter plots presented in Fig. 4 illustrate the change in the elemental distribution correlation in one tomographic reconstructed slice compared to a 2D projection. The entire cell was segmented into three compartments: the non-infected part of the cell, the parasitic vacuole, and the hemozoin crystals. We applied simple linear regression modeling to analyze the correlation between Fe and $\mathrm{S}$ in each compartment (see Table S1). The correlation coefficients in the hemozoin crystal part increased from 0.12 in the $2 \mathrm{D}$ projection to 0.33 in the tomographic reconstructed slice, yielding statistically relevant slope of 0.24 ( $\mathrm{p}$ value $=7.6 \times 10^{-5}$ ) between $\mathrm{Fe}$ and $\mathrm{S}$. 
Table 1: Elemental mass concentration and mass fraction

\begin{tabular}{lllllll} 
& \multicolumn{2}{l}{$\begin{array}{l}\text { mass concentration } \\
\left(\mu \mathrm{g} / \mathrm{mm}^{3}\right)\end{array}$} & $\begin{array}{l}\text { mass fraction } \\
(1 / 1000)\end{array}$ & $\begin{array}{l}\text { total mass } \\
(\mathrm{g})\end{array}$ & \\
\cline { 2 - 6 } & parasite & whole cell & parasite & whole cell & parasite & whole cell \\
$\mathrm{Fe}$ & 1.93 & 0.99 & 1.75 & 0.95 & $3.99 \times 10^{-14}$ & $8.29 \times 10^{-14}$ \\
$\mathrm{~S}$ & 12.52 & 8.75 & 11.19 & 8.43 & $2.63 \times 10^{-13}$ & $6.92 \times 10^{-13}$ \\
$\mathrm{P}$ & 22.40 & 10.48 & 20.28 & 10.07 & $3.42 \times 10^{-13}$ & $5.43 \times 10^{-13}$ \\
Mass density & $1.06 \times 10^{3}$ & $1.04 \times 10^{3}$ & & & $1.09 \times 10^{-11}$ & $2.74 \times 10^{-11}$
\end{tabular}
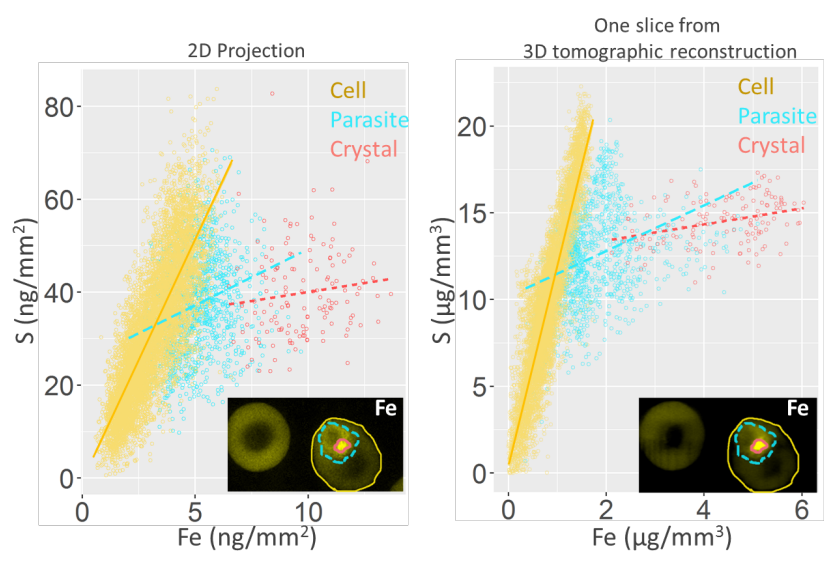

Figure 4: Scatter plots of $\mathrm{S}$ and Fe elemental maps in the infected red blood cell calculated on a 2D projection (left) and on a tomographic slice (right). The cell shown in the inset box was splitted manually into three major compartments: the cell (yellow), the parasite (cyan), and the hemozoin crystal (pink). These compartments correspond to the three clusters in the scatter plots. Linear regression lines are shown with the same color. The reconstructed tomography slice showed better linear relationships than the 2D projection, particularly in the cell body part.

\section{Conclusion}

We presented a workflow of correlative imaging with X-ray fluorescence tomography and phase contrast nanotomography to produce 3D mass concentration, mass density and mass fraction volumes. The approach was applied on malariainfected human red blood cells. Data processing was optimized particularly with respect to the reconstruction method used in fluorescence tomography. Using systematic evaluations of the resolution, the SNR and the fidelity, we found TVM with positivity constraint to give the most satisfactory results. High resolution intracellular 3D distributions of the key endogenous elements $\mathrm{Fe}, \mathrm{P}$ and $\mathrm{S}$ have clearly revealed the different cellular compartments, in particular the parasitic vacuole and the hemozoin crystals. Data analysis of the elemental correlations was shown to be more reliable on the reconstructed volumes compared to standard 2D projections. The quantitative analysis of the number of Fe atoms and the total cell mass proved to be in a very good agreement with previously reported values. Finally, the correlative nanotomography approach provided for the first time 3D mass fraction volumes of multiple elements.

Cryogenic preservation of the biological specimens is an important next step to maintain the cells in their close-to-native state, thus reducing chemical contamination and improving morphological stability. Combined with ever brighter X-ray nanoprobes, faster detector technology and improved reconstruction methods, correlative X-ray nanotomography is becoming the tool of choice for 3D analytical imaging of entire cells.

\section{References}

(1) Finney, L. A.; O'halloran, T. V. Science 2003, 300, 931-936.

(2) Janssens, K. H.; Adams, F.; Rindby, A. Microscopic X-ray fluorescence analysis; Wiley Chichester, 2000.

(3) Fahrni, C. J. Current opinion in chemical biology 2007, 11, 121-127.

(4) Yan, H.; Nazaretski, E.; Lauer, K.; Huang, X.; Wagner, U.; Rau, C.; 
Yusuf, M.; Robinson, I.; Kalbfleisch, S.; Li, L.; Bouet, N.; Zhou, J.; R., C.; Chu, Y. S. Scientific reports 2016, 6, 20112.

(5) Ice, G. E.; Budai, J. D.; Pang, J. W. Science 2011, 334, 1234-1239.

(6) da Silva, J. C.; Pacureanu, A.; Yang, Y.; Bohic, S.; Morawe, C.; Barrett, R.; Cloetens, P. Optica 2017, 4, 492-495.

(7) de Jonge, M. D.; Holzner, C.; Baines, S. B.; Twining, B. S.; Ignatyev, K.; Diaz, J.; Howard, D. L.; Legnini, D.; Miceli, A.; McNulty, I.; Jacobsen, C. J.; Vogt, S. Proceedings of the National Academy of Sciences 2010, 10\%, 15676-15680.

(8) Yuan, Y.; Chen, S.; Paunesku, T.; Gleber, S. C.; Liu, W. C.; Doty, C. B.; Mak, R.; Deng, J.; Jin, Q.; Lai, B.; Brister, K.; Flachenecker, C.; Jacobsen, C.; Vogt, S.; Woloschak, G. E. ACS Nano 2013, 7, 10502-10517, PMID: 24219664.

(9) Xu, F.; Helfen, L.; Suhonen, H.; Elgrabli, D.; Bayat, S.; Reischig, P.; Baumbach, T.; Cloetens, P. PLoS One 2012, \%, e50124.

(10) Victor, T. W.; Easthon, L. M.; Ge, M.; OToole, K. H.; Smith, R. J.; Huang, X.; Yan, H.; Allen, K. N.; Chu, Y. S.; Miller, L. M. Scientific reports 2018, 8, 13415 .

(11) Deng, J.; Lo, Y. H.; Gallagher-Jones, M.; Chen, S.; Pryor, A.; Jin, Q.; Hong, Y. P.; Nashed, Y. S. G.; Vogt, S.; Miao, J.; Jacobsen, C. Science Advances 2018, 4.

(12) Kosior, E.; Bohic, S.; Suhonen, H.; Ortega, R.; Devès, G.; Carmona, A.; Marchi, F.; Guillet, J. F.; Cloetens, P. Journal of structural biology 2012, 177, 239-247.

(13) Gramaccioni, C. et al. Applied Physics Letters 2018, 112, 053701.
(14) Grimshaw, K.; Sahler, J.; Spinelli, S. L.; Phipps, R. P.; Blumberg, N. Transfusion 2011, 51, 874-880.

(15) Kapishnikov, S.; Leiserowitz, L.; Yang, Y.; Cloetens, P.; Pereiro, E.; Ndonglack, F. A.; Matuschewski, K.; Als-Nielsen, J. Scientific reports 2017, 7, 802 .

(16) Phillips, K. G.; Jacques, S. L.; McCarty, O. J. Physical review letters 2012, 109, 118105.

(17) Mokso, R.; Cloetens, P.; Maire, E.; Ludwig, W.; Buffière, J.-Y. Applied physics letters 2007, 90, 144104.

(18) Adams, F.; Vekemans, B.; Silversmit, G.; De Samber, B.; Vincze, L. Handbook of nuclear chemistry; Springer, 2011; pp 1737-1759.

(19) Howells, M. R.; Hitchcock, A. P.; Jacobsen, C. J. Journal of Electron Spectroscopy and Related Phenomena 2009, 1, 1-3.

(20) Cloetens, P.; Ludwig, W.; Baruchel, J.; Van Dyck, D.; Van Landuyt, J.; Guigay, J.; Schlenker, M. Applied physics letters 1999, 75, 2912-2914.

(21) Mirone, A.; Brun, E.; Gouillart, E.; Tafforeau, P.; Kieffer, J. Nuclear Instruments and Methods in Physics Research Section B: Beam Interactions with Materials and Atoms 2014, 324, 41-48.

(22) Bracewell, R. N.; Riddle, A. The Astrophysical Journal 1967, 150, 427.

(23) Hsieh, J. Computed tomography: principles, design, artifacts, and recent advances. 2009.

(24) Fournet, G.; Guinier, A. Translated by Walker, CB and Yudowitch, KL In: New York: John Wiley \& Sons 1955, 7-78.

(25) Solé, V.; Papillon, E.; Cotte, M.; Walter, P.; Susini, J. Spectrochimica Acta Part B: Atomic Spectroscopy 2007, 62, 63-68. 
(26) MessaoudiI, C.; Boudier, T.; Sorzano, C. O. S.; Marco, S. BMC bioinformatics 2007, 8, 288.

(27) Dines, K. A.; Lytle, R. J. Proceedings of the IEEE 1979, 67, 1065-1073.

(28) Candès, E. J.; Romberg, J.; Tao, T. IEEE Transactions on information theory 2006, 52, 489-509.

(29) Shepp, L. A.; Vardi, Y. IEEE transactions on medical imaging 1982, 1, 113-122.

(30) De Nolf, W.; Vanmeert, F.; Janssens, K. Journal of Applied Crystallography 2014, 47, 1107-1117.

(31) Gürsoy, D.; De Carlo, F.; Xiao, X.; Jacobsen, C. Journal of synchrotron radiation 2014, 21, 1188-1193.

(32) Mattes, D.; Haynor, D. R.; Vesselle, H.; Lewellen, T. K.; Eubank, W. IEEE transactions on medical imaging 2003, 22, 120-128.

(33) Avants, B. B.; Tustison, N.; Song, G. Insight $j$ 2009, 2, 1-35.

(34) Dubar, F.; Bohic, S.; Slomianny, C.; Morin, J.-C.; Thomas, P.; Kalamou, H.; Guérardel, Y.; Cloetens, P.; Khalife, J.; Biot, C. Chemical Communications 2012, 48, 910-912.

(35) Mauritz, J. M.; Seear, R.; Esposito, A.; Kaminski, C. F.; Skepper, J. N.; Warley, A.; Lew, V. L.; Tiffert, T. Biophysical journal 2011, 100, 1438-1445.

(36) Pagot, E.; Fiedler, S.; Cloetens, P.; Bravin, A.; Coan, P.; Fezzaa, K.; Baruchel, J.; Härtwig, J. Physics in Medicine \& Biology 2005, 50, 709.

\section{Acknowledgement}

\section{Supporting Information Avail- able}

The supporting materials provide: (i) Movies of $\mathrm{X}$-ray fluorescence tomographic reconstructions on the elements $\mathrm{Fe}, \mathrm{S}$ and $\mathrm{P}$ in the red blood cells using 5 different tomography algorithms; (ii) Movie of X-ray phase contrast nanotomography of the malaria-infected red blood cell; (iii) Document on experimental setup, dose calculation and cross correlation estimation. 